\title{
Awe: Efeitos Cognitivos, Emocionais e Motivacionais do Deslumbramento num Contexto Experimental
}

\author{
Maria Eduarda Ferreira ${ }^{1, *}$ (D, Paulo Mendes ${ }^{1}$ (D, Beatriz Sardenberg ${ }^{1}$ (D, \\ \& Marina Martorelli Pinho ${ }^{1,2}$ (D) \\ ${ }^{1}$ Universidade Federal do Rio de Janeiro, Rio de Janeiro, RJ, Brasil \\ ${ }^{2}$ Centro Universitário Celso Lisboa, Rio de Janeiro, RJ, Brasil
}

\begin{abstract}
RESUMO - A relativamente inexplorada emoção de awe, que pode ser descrita em português como a sensação de deslumbramento e transcendência perante a percepção subjetiva de grandiosidade, vem sendo experimentalmente associada a efeitos positivos de natureza diversa. Nos artigos encontrados para inclusão nesta revisão bibliográfica, foram relatados um aumento da pró-socialidade e de sentimentos afiliativos, melhoras no humor e na satisfação pessoal, e modificações na concepção dos participantes acerca do tempo, levando a uma redução da impaciência e incentivando a busca de experiências de crescimento pessoal em lugar de recompensas imediatas. Esse sentimento também pareceu encorajar o senso crítico com relação a tentativas de convencimento, além de promover a espiritualidade e a identificação de propósito na realidade.
\end{abstract}

PALAVRAS-CHAVE: emoção, psicologia experimental, psicologia positiva, psicologia ambiental, revisão de literatura

\section{Awe: Cognitive, Emotional and Motivational Effects of Dazzle in an Experimental Context}

\begin{abstract}
The relatively uninvestigated emotion of awe has been experimentally associated with a wide range of positive effects. The articles retrieved for inclusion in this literature review reported an increase in prosociality and group affiliation, improvements in mood and personal satisfaction, and alterations in subjects' conception of time which reduced feelings of impatience and enhanced their disposition to seek personal growth experiences over immediate rewards. This emotion also seemed to encourage critical thinking with regard to persuasion attempts, and to promote spirituality as well as the identification of purpose in reality.
\end{abstract}

KEYWORDS: emotion, experimental psychology, positive psychology, environmental psychology, literature review

Apalavra inglesa awe, ainda sem tradução satisfatoriamente abrangente para o português, designa o sentimento evocado pela contemplação de um acontecimento ou cenário tão grandioso que altere, ainda que transitoriamente, a percepção do indivíduo sobre o mundo e sua própria posição nele (Keltner \& Haidt, 2003). Permeando do temor religioso ao deslumbramento com uma vasta paisagem natural e descrito desde escrituras sagradas hindus até a sociologia de Max Weber (citado por Keltner \& Haidt, 2003), o awe é uma sensação distintamente humana e universal, ainda que vivenciada de maneira íntima. No entanto, o assunto foi pouco explorado pela psicologia experimental até os dias de hoje, numa trajetória de início recente - o experimento mais antigo encontrado nas bases consultadas data de 2008 .
Talvez essa relativa carência de publicações se deva ao desafio inerente à realização de qualquer tipo de pesquisa científica dessa natureza: como resumir uma experiência subjetiva transcendente, aparentemente inacessível à comunicação mundana, na forma de um constructo bem definido e decomponível em seus elementos constituintes, passível de medição e análise estatística? Mais do que isso, como induzir, através de manipulação experimental, um estado pessoal e introspectivo por natureza, de modo a permitir a testagem através de escalas aplicadas antes e depois ou de tarefas comportamentais, a comparação com um grupo controle, e a realização de inferências válidas quanto à causalidade das correlações encontradas? Apesar da inegável dificuldade, os estudos experimentais realizados

\footnotetext{
*E-mail: ferreira.mev@gmail.com

- Submetido: 19/09/2016; Revisado: 18/07/2019; Aceito: 16/01/2020.
} 
nos últimos anos acerca do awe vêm progredindo na criação de instrumentos para estudá-lo e os resultados obtidos têm sido animadores, de modo a estimular a continuidade dos esforços. Esse sentimento já foi associado a efeitos positivos sobre diversas esferas centrais da vida humana, como o humor (Joye \& Bolderdijk, 2014) e a disposição para colaborar com os demais (Rudd et al., 2012; Piff et al., 2015; Joye \& Bolderdijk, 2014). Ao que tudo indica, trata-se de um tópico dotado de ricas possibilidades de estudo sobretudo para a psicologia positiva, podendo até mesmo dar origem a formas de aplicação e intervenção em campos diversos, caso se confirmem as associações entre awe, bem-estar e qualidade de vida encontradas até o momento - uma relação de causalidade que somente a pesquisa experimental pode comprovar.

Este artigo é o resultado da reunião e comparação do que vem sendo publicado no âmbito da psicologia experimental sobre a emoção de awe. Ele busca divulgar resultados e aprimorar os desenhos experimentais empregados para a exploração desse tema ainda incipientemente trabalhado, mas que pode encerrar não apenas um grande potencial de auxílio à compreensão da experiência humana, de maneira transcultural e atemporal, como também uma chave para o ganho de qualidade de vida em meio às atribulações do dia a dia contemporâneo.

\section{MÉTODO}

Os artigos para revisão foram pesquisados nas bases de dados Scielo, PubMed, EBSCO Academic Search Premier e APA PsycNET, empregando-se os descritores "awe" e "psychology" integrados pelo operador booleano "AND". Não houve restrição de idioma e a busca foi realizada até maio de 2016, incluindo artigos publicados até a ocasião.

Os critérios previamente estabelecidos para a inclusão foram a existência de algum tipo de contraste entre ao menos dois grupos de participantes, quer fosse com relação à pontuação numa escala ou à realização de uma tarefa comportamental, tendo um deles sido previamente submetido a alguma espécie de manipulação experimental para indução de awe, enquanto os demais estivessem sujeitos a outro estado emocional controle. Foram excluídos estudos que envolvessem apenas a realização de entrevistas ou a aplicação de questionários de maneira não vinculada a um contexto experimental indutor de awe.

Foram encontrados 502 artigos nas bases bibliográficas pesquisadas. Uma avaliação dos títulos e resumos resultou na exclusão de 487 artigos, ou por não tratarem do tema, ou por se tratar de resenhas de livros, artigos sobre a prática clínica, artigos de opinião, ou explorações apenas teóricas sobre a natureza do sentimento de awe. Dos 15 artigos restantes, 7 foram excluídos por envolverem apenas a aplicação de questionários sem a presença de uma intervenção experimental, não atendendo aos critérios de elegibilidade. A Figura 1 traz uma representação gráfica do processo descrito.

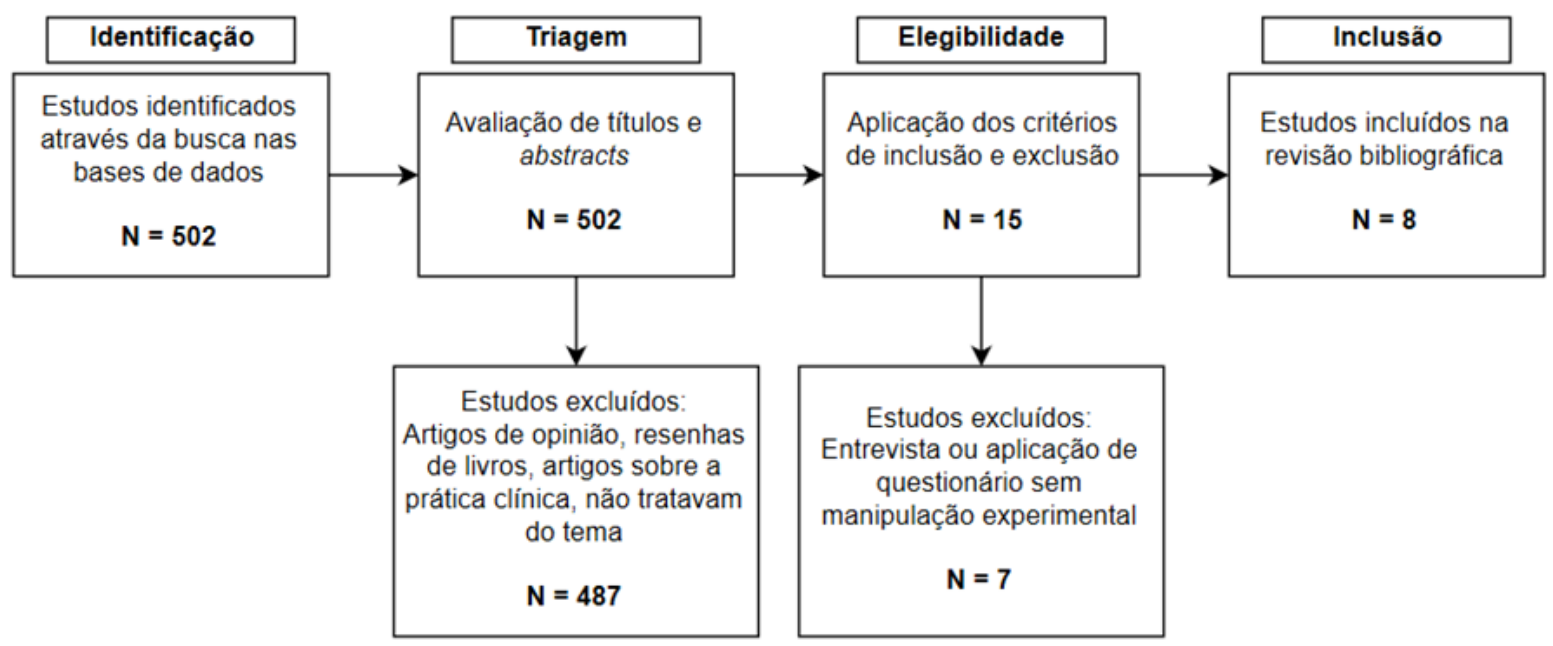

Figura 1. Fluxograma indicando o processo de identificação e seleção de artigos.

\section{RESULTADOS}

Os seis estudos experimentais e os dois estudos quaseexperimentais restantes após as exclusões detalhadas na seção "Método" passaram a compor esta revisão.
Talvez o resultado mais repetidamente relatado em experimentos e quase-experimentos acerca da emoção de awe seja um aumento da disposição para o comportamento 
pró-social (Joye \& Bolderdijk, 2014; Piff et al., 2015; Rudd et al., 2012), possivelmente associada a uma intensificação de sentimentos afiliativos (Cappellen \& Saroglou, 2012). Foi também descrito por mais de um artigo (Joye \& Bolderdijk, 2014; Rudd et al., 2012) um efeito de melhora no humor dos participantes ou em sua satisfação com sua própria vida após a evocação de awe. Ademais, esse estado emocional parece alterar a concepção dos sujeitos sobre o tempo, fazendo com que este pareça menos escasso e aqueles se tornem menos impacientes, além de mudar as prioridades dos indivíduos com relação à alocação desse recurso, levando-os a priorizar experiências vivenciais acima de bens materiais (Rudd et al., 2012), e experiências espirituais sobre outras de natureza hedonista (Cappellen \& Saroglou, 2012).

Curiosamente, a emoção estudada promove o que poderia ser descrito como um aumento do senso crítico com relação à avaliação de argumentos, tornando os participantes menos inclinados a se deixar persuadir por argumentação fracamente embasada (Griskevicius et al., 2010), mas isso não se refletiu num aumento do ceticismo com relação a temas de natureza mística: em Valdesolo e Graham (2014), pelo contrário, foi encontrada uma correlação entre a indução de awe e a interpretação de eventos como resultantes de uma determinação intencional e dirigida para um propósito (agency detection, ou deteç̧ão de agência, numa tradução livre) por parte dos sujeitos, medida através de escalas sobre a crença no sobrenatural e pela identificação de padrões (falsos) em sequências numéricas aleatórias.

Dois dos artigos incluídos, por fim, concentrando-se sobre as condições de evocação do sentimento de awe, identificaram uma maior predisposição a essa emoção em pessoas que pontuavam mais alto em escalas para medição do grande fator de personalidade Abertura à experiência (Silvia et al., 2015), e um maior potencial de eliciação desse estado por espaços naturais "selvagens" ou largamente intocados, em oposição àqueles visivelmente planejados e modificados pelo homem (Davis \& Gatersleben, 2013).

Os métodos empregados na evocação do sentimento de awe foram diversos, incluindo a exposição a imagens ou vídeos de grandiosidade na natureza, como tornados ou o pôr do sol, a produção de relatos sobre vivências anteriores dessa sensação como maneira de provocá-la novamente através da concentração na memória do evento, e a exposição à natureza in vivo. Todos os experimentos se valeram da indução de outras emoções positivas e/ou de estados emocionais neutros em condições similares, como situações controle.

A Tabela 1 apresenta um detalhamento do método e resultados de cada artigo individualmente.

\section{DISCUSSÃO}

Todos os estudos incluídos na revisão apontam para efeitos positivos de experiências indutoras de awe sobre os sujeitos, o que talvez não seja de todo surpreendente. Tal simplicidade é apenas aparente: uma análise aprofundada dos resultados em conjunto revela não só implicações além da simples confirmação da crença, algo intuitivo, de que esses estados emocionais sejam agradáveis, como também acaba por gerar mais perguntas do que respostas, apontando diversos possíveis caminhos para a recém-iniciada exploração desse tema pela psicologia experimental.

Uma dimensão em que foram feitas observações aparentemente contrastantes é a maneira como o awe impacta a relação de um indivíduo com os demais. Se, por um lado, a indução de awe foi associada à intensificação da sensação de pertencimento tanto a um grupo de pessoas próximas quanto à coletividade ou humanidade de maneira geral (Capellen \& Saroglou, 2012), e repetidamente mostrou-se um fator de incentivo ao comportamento prósocial (Joye \& Bolderdijk, 2014; Piff et al., 2015; Rudd et al., 2012). Por outro lado, não parece haver qualquer correlação entre a Extroversão - conforme definida pelo modelo dos cinco grandes fatores de personalidade - e a propensão a vivenciar emoções associadas ao awe. Esse resultado ocorre a despeito da reconhecida relação entre esse traço e a ocorrência de estados emocionais positivos de maneira geral (Silvia et al., 2015), e da própria definição do fator Extroversão como caracterizado pela predisposição a interagir com outras pessoas (Ashton \& Lee, 2001). Pode ser notado também que as diferentes estratégias de manipulação experimental empregadas para gerar awe nos participantes foram todas atividades individuais, nas quais muitas vezes sequer havia qualquer espécie de referência à socialização ou à presença de outras pessoas: no caso dos estudos que se valeram de fotografias de cenários naturais, por exemplo, não foi relatada por nenhum dos autores a escolha preferencial por imagens em que houvesse pessoas presentes (Joye \& Bolderdijk, 2014; Silvia et al., 2015; Piff et al., 2015).

Extrai-se daí que o awe é, simultaneamente, uma emoção que não tem um caráter inerentemente social - isto é, que não depende de um contexto de interação interpessoal para ser eliciada e que, na verdade, talvez seja mais facilmente evocada por experiências introspectivas, que muitas vezes ocorrem de maneira solitária - e um modificador da maneira como uma pessoa se vê com relação às demais. Devido à ausência de um componente social direto nesse tipo de experiência, é razoável supor que esse impacto seja consequência de influências mais amplas do awe sobre a cognição. Piff et al. (2015) observaram uma correlação entre a indução experimental de awe e o fenômeno denominado small self ("eu pequeno", numa tradução literal), em que o indivíduo vivencia uma diminuição subjetiva da percepção de seu próprio eu e seus desejos e objetivos face à consciência de uma entidade tida como maior e mais poderosa. Mais do que isso, uma análise de mediação confirmou que os efeitos 
Tabela 1

Artigos incluidos na revisão

\begin{tabular}{|c|c|c|c|}
\hline Autor/Ano & Título & Delineamento & Resultados \\
\hline $\begin{array}{l}\text { Davis e } \\
\text { Gatersleben } \\
(2013)\end{array}$ & $\begin{array}{l}\text { Transcendent Experiences in } \\
\text { Wild and Manicured Settings: } \\
\text { The Influence of the Trait } \\
\text { 'Connectedness to Nature' }\end{array}$ & $\begin{array}{l}\text { Estudo quase-experimental }(\mathrm{n}=253) \text { com } \\
\text { aplicação de questionários sobre estado } \\
\text { emocional a dois grupos após um intervalo } \\
\text { de tempo passado em ambiente selvagem ou } \\
\text { modificado pelo homem. }\end{array}$ & $\begin{array}{l}\text { Awe caracterizado como mais fortemente } \\
\text { induzido por ambientes selvagens, enquanto o } \\
\text { contato com a natureza modificada produziria } \\
\text { mais sentimentos de calma. }\end{array}$ \\
\hline $\begin{array}{l}\text { Joye e } \\
\text { Bolderdijk } \\
(2014)\end{array}$ & $\begin{array}{l}\text { An exploratory study into } \\
\text { the effects of extraordinary } \\
\text { nature on emotions, mood, and } \\
\text { prosociality }\end{array}$ & $\begin{array}{l}\text { Estudo quase-experimental }(\mathrm{n}=202) \mathrm{em} \\
\text { que três grupos assistiam em casa, pela } \\
\text { internet, a apresentações de slides contendo } \\
\text { imagens indutoras ou não de awe. }\end{array}$ & $\begin{array}{l}\text { Awe associado a melhoras no humor e ao } \\
\text { aumento do escore de orientação de valor social } \\
\text { (SVO) numa tarefa de alocação de recursos. } \\
\text { Nenhum impacto sobre a disposição para fazer } \\
\text { doações. }\end{array}$ \\
\hline $\begin{array}{l}\text { Rudd, Vohs e } \\
\text { Aaker (2012) }\end{array}$ & $\begin{array}{l}\text { Awe Expands People's } \\
\text { Perception of Time, Alters } \\
\text { Decision Making, and Enhances } \\
\text { Well-Being }\end{array}$ & $\begin{array}{l}\text { Estudo incluindo três experimentos } \\
\text { randomizados comparando condições } \\
\text { indutoras de felicidade e awe }(\mathrm{n}=254) \text {. }\end{array}$ & $\begin{array}{l}\text { Avaliação do tempo como mais abundante, } \\
\text { menor impaciência, maior disposição para doar } \\
\text { tempo por meio de trabalho voluntário (mas não } \\
\text { dinheiro), aumento da satisfação com a própria } \\
\text { vida e priorização de experiências prazerosas em } \\
\text { oposição a bens materiais na condição } a w e .\end{array}$ \\
\hline $\begin{array}{l}\text { Piff, Dietze, } \\
\text { Feinberg, } \\
\text { Stancato } \\
\text { e Keltner } \\
(2015)\end{array}$ & $\begin{array}{l}\text { Awe, the Small Self, and } \\
\text { Prosocial Behavior }\end{array}$ & $\begin{array}{l}\text { Estudo incluindo cinco experimentos } \\
\text { randomizados induzindo diferentes } \\
\text { sentimentos, incluindo awe, ou participantes } \\
\text { com variações prévias na frequência com } \\
\text { que vivenciam o sentimento de awe em sua } \\
\text { vida diária }(\mathrm{n}=2078) \text {. }\end{array}$ & $\begin{array}{l}\text { Correlação entre frequência prévia de } \\
\text { experiências de awe e generosidade num jogo } \\
\text { econômico. A indução de awe levou a uma maior } \\
\text { ética e generosidade na tomada de decisões, } \\
\text { mediada pela sensação de insignificância ou } \\
\text { conexão a algo maior (small self). Os resultados } \\
\text { se confirmaram mesmo quando o awe não estava } \\
\text { associado à natureza, ou estava associado ao } \\
\text { medo. }\end{array}$ \\
\hline $\begin{array}{l}\text { Valdesolo } \\
\text { e Graham } \\
(2014)\end{array}$ & $\begin{array}{l}\text { Awe, Uncertainty, and Agency } \\
\text { Detection }\end{array}$ & $\begin{array}{l}\text { Estudo incluindo cinco experimentos } \\
\text { randomizados de manipulação de awe em } \\
\text { comparação a uma condição neutra e, em } \\
\text { alguns casos, humor positivo inespecífico } \\
(\mathrm{n}=484) \text {. }\end{array}$ & $\begin{array}{l}\text { Correlação entre awe induzido e: crença em } \\
\text { forças sobrenaturais, incluindo o controle } \\
\text { sobrenatural dos acontecimentos; desconforto } \\
\text { com a ambiguidade; e percepção de uma } \\
\text { organização intencional em padrões numéricos } \\
\text { aleatórios. }\end{array}$ \\
\hline $\begin{array}{l}\text { Griskevicius, } \\
\text { Shiota e } \\
\text { Neufeld } \\
(2010)\end{array}$ & $\begin{array}{l}\text { Influence of Different Positive } \\
\text { Emotions on Persuasion } \\
\text { Processing: A Functional } \\
\text { Evolutionary Approach }\end{array}$ & $\begin{array}{l}\text { Estudo incluindo dois experimentos } \\
\text { randomizados em que, após a evocação } \\
\text { de diferentes emoções, inclusive awe, os } \\
\text { participantes }(\mathrm{n}=735 \text { ) eram expostos a } \\
\text { mensagens persuasivas com argumentação } \\
\text { forte ou fracamente embasada. }\end{array}$ & $\begin{array}{l}\text { Correlação entre o sentimento de awe e uma } \\
\text { menor propensão a se deixar persuadir por } \\
\text { argumentação de teor fraco. }\end{array}$ \\
\hline $\begin{array}{l}\text { Cappellen } \\
\text { e Saroglou } \\
(2012)\end{array}$ & $\begin{array}{l}\text { Awe Activates Religious } \\
\text { and Spiritual Feelings and } \\
\text { Behavioural Intentions }\end{array}$ & $\begin{array}{l}\text { Estudo com dois experimentos } \\
\text { randomizados de indução de awe ou de um } \\
\text { controle }(\mathrm{n}=219) \text {, seguida de questionário } \\
\text { sobre o sentimento de unidade com os } \\
\text { demais ou da escolha hipotética por um } \\
\text { local de viagem. }\end{array}$ & $\begin{array}{l}\text { O estado de awe promove a sensação de } \\
\text { comunhão com um grupo de amigos e favorece } \\
\text { a escolha pela realização de uma viagem } \\
\text { espiritual, em vez de uma opção hedonista, em } \\
\text { pessoas religiosas (efeito não significativo em } \\
\text { pessoas não religiosas). }\end{array}$ \\
\hline $\begin{array}{l}\text { Silvia, Fayn, } \\
\text { Nusbaum e } \\
\text { Beaty }(2015)\end{array}$ & $\begin{array}{l}\text { OTE and Awe in Response to } \\
\text { Nature and Music: Personality } \\
\text { and Profound Aesthetic } \\
\text { Experiences }\end{array}$ & $\begin{array}{l}\text { Estudo experimental de duas fases }(\mathrm{n}=103) \\
\text { envolvendo a avaliação dos sujeitos, em um } \\
\text { questionário, da experiência de visualizar } \\
\text { imagens do Universo e escutar uma canção } \\
\text { indutora de awe. }\end{array}$ & $\begin{array}{l}\text { Correlação entre o fator de personalidade } \\
\text { Abertura à experiência e tendência a vivenciar } \\
\text { awe; correlações menores encontradas para } \\
\text { outros fatores, exceto Extroversão. }\end{array}$ \\
\hline
\end{tabular}

sobre a generosidade dos participantes nas tarefas propostas estavam muito mais relacionados à intensidade com que o awe havia evocado a sensação de small self do que com o quão fortemente o awe em si havia sido vivenciado.

Nenhum dos outros artigos selecionados empregou medidas para avaliar o efeito small self, de modo que essa mediação encontrada preliminarmente necessita de mais estudos para que seja confirmada e explorada mais a fundo. No entanto, o modelo proposto é condizente com o entendimento de que o awe é evocado pela percepção de uma grandiosidade que exceda as dimensões das estruturais mentais existentes a ponto de exigir acomodação para que possa ser assimilada (Keltner \& Haidt, 2003): se uma experiência de awe de alguma forma leva o indivíduo a operar numa "escala" maior, é possível que as dimensões de seu próprio eu passem a não mais ocupar uma parcela 
tão grande de sua percepção da realidade, possibilitando que ele enxergue com mais facilidade e leve em consideração as necessidades e desejos dos demais, além de ressaltar a semelhança destes com os seus (o que pode ser uma causa para a sensação de pertencimento aumentada, descrita por Capellen \& Saroglou, 2012).

Esse processo de acomodação pode estar também na base da mudança de perspectiva com relação ao tempo observada por Rudd et al. (2012), em que sujeitos que haviam sentido awe, em comparação com outra emoção positiva (felicidade) ou uma condição neutra, pontuaram mais baixo numa escala para medição do sentimento de impaciência e mais alto numa escala de avaliação da crença pessoal na disponibilidade ampla de tempo. Da mesma maneira como um indivíduo pode se ver numa dimensão menor com relação a outras pessoas, ele talvez passe a ter em mente, de maneira consciente ou não, uma escala de tempo maior, como a duração da vida de uma pessoa ou mesmo o tempo histórico, geológico ou cósmico, de modo que o momento atual pareça menor e menos significativo, e o tempo como um todo pareça mais vasto.

Essa mudança de escala temporal traz possíveis implicações sobre a motivação ainda não exploradas experimentalmente. No mesmo artigo mencionado acima (Rudd et al., 2012), foi demonstrado que o awe torna uma pessoa mais disposta a dedicar seu tempo a uma atividade voluntária - e não a fazer doações em dinheiro, o que aponta para um impacto ao menos parcial da mudança na percepção do tempo sobre essa disposição, além do simples aumento da orientação de valor social observado - e a escolher uma experiência futura agradável, em vez de uma recompensa material de valor equivalente. Um efeito possivelmente relacionado foi relatado por Capellen e Saroglou (2012), que observaram uma maior tendência, por parte de participantes religiosos em que a emoção de awe havia sido provocada, de escolha por uma viagem hipotética a um destino previamente classificado pelos respondentes como espiritual (Tibet) mesmo quando oferecida a opção de visitar outro local (Haiti) anteriormente associado ao lazer ou ao hedonismo. É digna de investigação a influência desses estados emocionais sobre a capacidade de se recusar uma gratificação imediata em prol de um benefício maior no futuro, que, se confirmada, poderia resultar em intervenções baseadas na indução de awe para o controle da impulsividade e de comportamentos compulsivos.

Essa possibilidade teórica também ganha força com base em estudos anteriores não relacionados ao awe que apontam para semelhanças neurológicas entre a maneira como são considerados os eus presente e futuro de uma pessoa, e a própria pessoa e outros indivíduos, num contexto de tomada de decisões (Jamison \& Wegener, 2010). Já foi observada uma correlação entre a tendência a abrir mão de benefícios para si em prol da colaboração com outras pessoas (quantificada por escores de orientação de valor social) e a realização de escolhas economicamente mais prudentes a longo prazo, como escolhas mais favoráveis ao "eu futuro" de um indivíduo em detrimento de seu "eu presente" (Silva et al., 2015). Tal resultado sugere que esse fenômeno possa depender do que poderia ser descrito como "empatia consigo mesmo", ou da capacidade de entender e levar em consideração seus próprios sentimentos e necessidades num momento futuro em que eles difiram ou até venham de encontro aos atuais. Sendo maiores índices de comportamento pró-social um dos efeitos mais consistente e repetidamente observados em estudos experimentais acerca do awe, é razoável imaginar um impacto semelhante sobre decisões com relação ao futuro.

Uma falha comum a todos os artigos considerados é terem sido realizados ao longo de um curto intervalo de tempo: os resultados dizem respeito apenas aos efeitos imediatos da manipulação experimental evocadora de awe. Ao menos até o momento da realização da busca nas bases citadas, não haviam sido feitos estudos em que os sujeitos fossem acompanhados após a manipulação por um período mais extenso, para que se pudesse observar por quanto tempo os efeitos do awe podem ser sentidos, ou se são rapidamente anulados pelas vivências cotidianas. Tampouco houve estudos em que a sensação de awe fosse induzida de maneira reiterada ao longo do tempo: o awe parece aumentar a satisfação subjetiva com a própria vida imediatamente após sua indução (Rudd et al., 2012), mas não se sabe se a inserção repetida do awe na vida de uma pessoa teria efeitos sobre seu bem-estar a longo prazo ou traria modificações comportamentais.

Devido à carência de avaliações sobre impactos de natureza duradoura, os indicadores de maior disposição a desempenhar ações específicas, como o trabalho voluntário, têm capacidade indeterminada de prever como os indivíduos testados efetivamente se comportam em suas vidas posteriormente à participação nos estudos, servindo apenas como indicadores do estado mental em que eles se encontravam logo após os experimentos. A realização de estudos longitudinais sobre o tema é essencial para avaliar a viabilidade e eficiência de intervenções baseadas em awe em psicoterapia ou na educação, por exemplo: os menores índices de convencimento por argumentação fracamente embasada entre participantes na condição awe relatados por Griskevicius et al. (2010) podem levar ao desenvolvimento de maior senso crítico com a repetição da experiência, ou trata-se de uma flutuação momentânea que não traria benefícios permanentes independentemente da duração ou frequência de uma intervenção? O caráter dos dados obtidos até o momento traz aplicações seguras apenas para áreas como a arquitetura, o paisagismo e o turismo, em que é importante a relação imediata estabelecida entre o indivíduo e o meio, sobretudo em sua dimensão estética.

Outra avaliação crítica a ser feita acerca dos estudos também digna de investigação mais aprofundada, sobretudo antes do desenvolvimento de aplicações práticas baseadas em seus resultados - é o quanto a variação individual 
observada no sucesso da indução de awe pode ter sido dependente de características prévias dos sujeitos, de modo que nem todas as pessoas possam ser igualmente beneficiadas por intervenções do gênero. É possível que os maiores beneficiados por elas sejam aqueles que já partem de uma linha de base mais elevada com relação aos fatores medidos e que, consequentemente, teriam menor necessidade real de uma intervenção.

Davis e Gatersleben (2013) observaram que um ambiente "selvagem", de natureza não modificada pelo homem, tinha, ao mesmo tempo, maior potencial indutor de awe, se comparado a um jardim cuidadosamente organizado, e maior probabilidade de provocar sentimentos negativos ou perturbadores em alguns indivíduos. Não houve correlação entre a presença ou intensidade do awe em si e a ocorrência desses sentimentos perturbadores, mas o achado chama atenção para o fato de que nem todas as pessoas reagem do mesmo modo ao mesmo tipo de ambiente, o que deve ser considerado no desenvolvimento de estratégias para induzir awe - não só visando a uma aplicação prática, como até mesmo para a realização de futuros experimentos, em que talvez devesse existir um maior controle das características pessoais dos participantes. Nesse estudo, o traço de personalidade connectedness to nature, medido através da escala de Mayer e Frantz (2004, citados por Davis \& Gatersleben, 2013), foi um preditor tanto da evocação de awe quanto de emoções perturbadoras, ainda que, por se tratar de um estudo quase-experimental, em que os próprios sujeitos escolheram o ambiente a ser visitado, não seja possível determinar se as diferenças observadas foram ao menos parcialmente afetadas por características ou fatores que tenham motivado a opção por um ou outro local, uma limitação reconhecida pelos próprios autores.

No que parece ter sido o único experimento do gênero até o momento, Silvia et al. (2015) avaliaram o impacto dos cinco grandes fatores de personalidade sobre a experiência de sentimentos associados ou não ao awe após a exposição a imagens do espaço sideral e a uma canção escolhida com base em trabalhos musicológicos anteriores. Todos os fatores de personalidade - exceto Extroversão, conforme já mencionado acima - previram ao menos uma das sensações avaliadas. Sujeitos com escores mais elevados de
Neuroticismo, por exemplo, tiveram maior probabilidade de relatar confusão após visualizar as imagens do espaço e de sentirem-se arrepiados com a música. Num efeito mais notável e talvez de maior significado, o fator Abertura à experiência teve não só correlações comparativamente mais fortes do que as observadas com os demais traços, como também esteve associado a todos ou quase todos os componentes da experiência de awe em ambas as condições.

Uma possível implicação disso é que uma pessoa que obtivesse uma pontuação baixa numa escala de Abertura à experiência, por exemplo, não se beneficiaria tanto quanto as demais de uma intervenção baseada em awe, sendo mais adequado buscar outras técnicas para obter os efeitos cognitivos ou comportamentais desejados, enquanto uma pessoa que pontue de maneira elevada nessa mesma escala poderia considerar essa mesma experiência intensamente proveitosa e agradável. Esse fator de personalidade deveria também ser controlado em experimentos posteriores, a fim de verificar se essa associação se sustentará em outros contextos.

Pode ser apontado como limitação deste artigo o fato de não se tratar de uma revisão sistemática e não terem sido considerados todos os artigos publicados sobre o tema awe na psicologia até hoje. No entanto, a opção por estudos experimentais (e quase-experimentais) foi feita tendo em mente que entrevistas ou questionários sem qualquer tipo de controle com relação às condições anteriores dos participantes poderiam gerar impactos imprevisíveis sobre os resultados obtidos. Além disso, estratégias desenvolvidas para a indução de awe num contexto experimental podem ser aproveitadas em outras situações em que se deseje aplicar esse conhecimento, associadas a áreas como o paisagismo ou a psicologia clínica.

Espera-se que o assunto awe continue sendo explorado na psicologia experimental, sobretudo por meio do desenvolvimento de novas metodologias de pesquisa com base nos resultados apresentados na literatura até agora, para que nosso entendimento acerca do tema seja expandido e tornado mais preciso, podendo inclusive dar origem a aplicações práticas que tragam ganhos de qualidade de vida para pessoas nas mais variadas situações.

\section{REFERÊNCIAS}

Ashton, M. C., \& Lee, K. (2001). A theoretical basis for the major dimensions of personality. European Journal of Personality, 15(5), 327-353. https://doi.org/10.1002/per.417

Cappellen, P. V., \& Saroglou, V. (2012). Awe activates religious and spiritual feelings and behavioral intentions. Psychology of Religion and Spirituality, 4(3), 223-236. https://doi. org/10.1037/a0025986

Davis, N., \& Gatersleben, B. (2013). Transcendent Experiences in Wild and Manicured Settings: The Influence of the Trait
"Connectedness to Nature". Ecopsychology, 5(2), 92-102. https://doi.org/10.1089/eco.2013.0016

Griskevicius, V., Shiota, M. N., \& Neufeld, S. L. (2010). Influence of different positive emotions on persuasion processing: A functional evolutionary approach. Emotion, 10(2), 190-206. https://doi.org/10.1037/a0018421

Jamison, J., \& Wegener, J. (2010). Multiple selves in intertemporal choice. Journal of Economic Psychology, 31(5), 832-839. https://doi.org/10.1016/j.joep.2010.03.004 
Joye, Y., \& Bolderdijk, J. W. (2015). An exploratory study into the effects of extraordinary nature on emotions, mood, and prosociality. Frontiers in Psychology, 5. https://doi. org/10.3389/fpsyg.2014.01577

Keltner, D., \& Haidt, J. (2003). Approaching awe, a moral, spiritual, and aesthetic emotion. Cognition \& Emotion, 17(2), 297-314. https://doi.org/10.1080/02699930302297

Piff, P. K., Dietze, P., Feinberg, M., Stancato, D. M., \& Keltner, D. (2015). Awe, the small self, and prosocial behavior. Journal of Personality and Social Psychology, 108(6), 883-899. https:// doi.org/10.1037/pspi0000018

Rudd, M., Vohs, K. D., \& Aaker, J. (2012). Awe expands people's perception of time, alters decision making, and enhances well- being. Psychological Science, 23(10), 1130-1136. https://doi. org/10.1177/0956797612438731

Silva, S. D., Matsushita, R., \& Carvalho, M. D. (2015). Prosocial people take better care of their own future well-being. OALib, 02(12), 1-7. https://doi.org/1010.4236/oalib.1102181

Silvia, P. J., Fayn, K., Nusbaum, E. C., \& Beaty, R. E. (2015). Openness to experience and awe in response to nature and music: Personality and profound aesthetic experiences. Psychology of Aesthetics, Creativity, and the Arts, 9(4), 376384. https://doi.org/10.1037/aca0000028

Valdesolo, P., \& Graham, J. (2013). Awe, uncertainty, and agency detection. Psychological Science, 25(1), 170-178. https://doi. org/10.1177/0956797613501884 